International Journal of Pure and Applied Mathematics

Volume 116 No. 1 2017, 197-200

ISSN: 1311-8080 (printed version); ISSN: 1314-3395 (on-line version)

url: http://www.ijpam.eu

doi: 10.12732/ijpam.v116i1.21

\title{
ON SOFT REGULARITY
}

\author{
M. Matejdes \\ Department of Mathematics and Computer Science \\ Faculty of Education, Trnava University in Trnava \\ Priemyselná 4, 91843 Trnava, SLOVAKIA
}

\begin{abstract}
The paper deals with two forms of soft regular separation axioms in soft topological spaces. Namely a characterization of two types of soft regular soft topological spaces via topology on Cartesian product is given. With regard to the proven results both regular soft topological spaces are quite trivial.
\end{abstract}

AMS Subject Classification: 54A05, 54D10

Key Words: soft set, soft topological space, soft separation axioms

\section{Introduction}

We recall the basic concept related to soft sets, soft topological spaces and soft separation axioms. For details see references.

Let $X, A$ be nonempty sets. A pair $(F, A)$ is called a soft set over $X$ with respect to a set of parameters $A$, where $F: A \rightarrow 2^{X}$ is a set valued mapping from $A$ to the power set $2^{X}$ of $X$. The soft union (soft intersection) of two soft sets $(F, A),(G, A)$, denoted by $(F, A) \tilde{\cup}(G, A)((F, A) \tilde{\cap}(G, A))$, is defined as a soft set $(H, A)$, where $H(e)=F(e) \cup G(e)(H(e)=F(e) \cap G(e))$ for all $e \in A$. (The soft union and intersection of a collection of soft sets is defined similarly). A soft set $(F, A)$ is a soft subset of $(G, A)$, denoted by $(F, A) \tilde{\subset}(G, A)$, if $F(e) \subset G(e)$ for all $e \in A$. A soft set $(F, A)$ is said to be a null (an absolute) soft set, denoted by $\Phi_{A}\left(X_{A}\right)$, if $F(e)=\emptyset(F(e)=X)$ for any $e \in A$. If a

$\begin{array}{lr}\text { Received: } & \text { July 7, } 2017 \\ \text { Revised: } & \text { August 21, 2017 } \\ \text { Published: } & \text { August 30, 2017 }\end{array}$

(c) 2017 Academic Publications, Ltd. url: www.acadpubl.eu 
collection $\tau$ of soft sets over $X$ with respect to a set of parameters $A$ contains the null soft set, the absolute soft set and $\tau$ is closed under arbitrary union and finite intersection of soft sets, then $\tau$ is called a soft topology and $(X, \tau, A)$ is called a soft topological space. A soft point, denoted by $e_{F}$, is a soft set $(F, A)$ for which $F(e) \neq \emptyset$ and $F\left(e_{1}\right)=\emptyset$ for $e_{1} \neq e$ and $e_{F} \tilde{\in}(G, A)(x \in(G, A))$ means $F(e) \subset G(e)(x \in G(e)$ for all $e \in A)$.

\section{Characterization of Soft Regularity}

A graph of a set valued mapping $F: A \rightarrow 2^{X}$ is the set $G r(F):=\{[e, x] \in$ $A \times X: x \in F(e)\}$ and it is a subset of $A \times X$. Denote $\operatorname{Dom}(F):=\{e \in$ $A: F(e) \neq \emptyset\}$ and $\operatorname{Ran}(F)=\cup_{e \in A} F(e)$. Since a set valued mapping can be understood as a subset of the Cartesian product $A \times X$, a soft set over $X$ with respect to a set of parameters $A$ can be introduced as a subset of $A \times X$. Consequently, the basic soft operations (the soft union, the soft intersection, the soft complement) can be defined by the corresponding set operations on the graphs of set valued mappings. For example, two soft sets $(F, A)$ and $(G, A)$ are soft disjoint if and only if $\operatorname{Gr}(F) \cap \operatorname{Gr}(G)=\emptyset,(F, A)$ is a soft subset of $(G, A)$ if and only if $\operatorname{Gr}(F) \subset G r(G)$. The absolute soft set (the null soft set) corresponds to the set $A \times X(\emptyset \subset A \times X)$.

As it has already been pointed out in [6], [7] a soft topological space is noting else as a topology on $A \times X$. More precisely, if $(X, \tau, A)$ is a soft topological space, then $\left(A \times X, \tau_{A \times X}\right)$ is a topological space, where $\tau_{A \times X}=\{G r(F)$ : $(F, A) \in \tau\}$. It is clear that for any $e \in A,\left(X, t_{e}\right)$ is a topological space, where $t_{e}=\{F(e):(F, A) \in \tau\}$. On the other hand, if $(A \times X, \sigma)$ is a topological space, then $(X, \tau, A)$ is a soft topological space, where $\tau=\left\{\left(F_{S}, A\right): S \in \sigma\right\}$ and $F_{S}: A \rightarrow 2^{X}, F_{S}(e)=\{x \in X:[e, x] \in S\}$ for all $e \in A$.

Definition 2.1. A soft topological space $(X, \tau, A)$ is said to be soft $\operatorname{regular}^{H}[5]$ (soft regular ${ }^{S}[9]$ ) if for any soft closed set $(G, A)$ and any $e_{F} \tilde{\notin}(G, A)$ $(x \notin(G, A))$, there exist soft open sets $\left(F_{1}, A\right),\left(F_{2}, A\right)$ such that $e_{F} \tilde{\in}\left(F_{1}, A\right)$ $\left(x \in\left(F_{1}, A\right)\right),(G, A) \tilde{\subset}\left(F_{2}, A\right)$ and $\left(F_{1}, A\right) \tilde{\cap}\left(F_{2}, A\right)=\Phi_{A}$.

Theorem 2.1. Let $(X, \tau, A)$ be a soft topological space. Then it is soft regular $^{H}$ if and only if for any soft closed set $(G, A), \operatorname{Gr}(G)=\operatorname{Dom}(G) \times X$ and a topological space $\left(A, \sigma_{\tau}\right)$ is regular, where $\sigma_{\tau}=\{\operatorname{Dom}(H):(H, A) \in \tau\}$.

Proof. " $\Rightarrow "$ Suppose $(X, \tau, A)$ is soft $\operatorname{regular}^{H}$ and $(G, A)$ is soft closed not equal to $\Phi_{A}$ (the equation $\operatorname{Gr}\left(\Phi_{A}\right)=\operatorname{Dom}\left(\Phi_{A}\right) \times X$ is clear). We prove $G(e)=X$ for any $e \in \operatorname{Dom}(G)$. Let $x \in X \backslash G\left(e^{0}\right)$ for some $e^{0} \in \operatorname{Dom}(G)$. 
Then there exists $y \neq x$ such that $y \in G\left(e^{0}\right)$. Define a set valued mapping $F$ as $F\left(e^{0}\right)=\{x, y\}$ and $F(e)=\emptyset$ for $e \neq e^{0}$. It is clear $e_{F}^{0} \tilde{\notin}(G, A)$. Since $(X, \tau, A)$ is soft $\operatorname{regular}^{H}$, there exist soft open sets $\left(F_{1}, A\right),\left(F_{2}, A\right)$ such that $e_{F}^{0} \tilde{\in}\left(F_{1}, A\right)$, $(G, A) \tilde{\subset}\left(F_{2}, A\right)$ and $\left(F_{1}, A\right) \tilde{\cap}\left(F_{2}, A\right)=\Phi_{A}$, which is a contradiction with $y \in$ $F_{1}\left(e^{0}\right) \cap F_{2}\left(e^{0}\right)$. So, if $(G, A)$ is soft closed (open), then $\operatorname{Gr}(G)=\operatorname{Dom}(G) \times X$. That means $\sigma_{\tau}$ is a topology on $A$.

We prove $\left(A, \sigma_{\tau}\right)$ is regular. Let $A_{0}$ be closed and $e \notin A_{0}$. Then there is a soft closed set $(G, A)$, such that $A_{0}=\operatorname{Dom}(G)$. Define a set valued mapping $F$ as $F(e)=X$ and $F\left(e^{\prime}\right)=\emptyset$ for $e^{\prime} \neq e$. Since $e_{F} \tilde{\notin}(G, A)$, there exist soft open sets $\left(F_{1}, A\right),\left(F_{2}, A\right)$ such that $e_{F} \tilde{\in}\left(F_{1}, A\right),(G, A) \tilde{\subset}\left(F_{2}, A\right)$ and $\left(F_{1}, A\right) \tilde{\cap}\left(F_{2}, A\right)=\Phi_{A}$. Since $G r\left(F_{i}\right)=\operatorname{Dom}\left(F_{i}\right) \times X$ for $i=1,2, \operatorname{Dom}\left(F_{1}\right) \cap$ $\operatorname{Dom}\left(F_{2}\right)=\emptyset, \operatorname{Dom}\left(F_{1}\right), \operatorname{Dom}\left(F_{2}\right)$ are open in $\sigma_{\tau}, e \in \operatorname{Dom}\left(F_{1}\right)$ and $A_{0} \subset$ $\operatorname{Dom}\left(F_{2}\right)$. So $\left(A, \sigma_{\tau}\right)$ is regular.

$" \Leftarrow "$ Let $(G, A)$ be a soft closed set and $e_{F} \tilde{\notin}(G, A)$. Since $e \notin \operatorname{Dom}(G)$ (otherwise $F(e) \subset G(e)=X$ ) and $\operatorname{Dom}(G)$ is closed, there are disjoint open sets $G_{1}, G_{2} \in \sigma_{\tau}$ such that $e \in G_{1}$ and $\operatorname{Dom}(G) \subset G_{2}$. Define $\left(F_{1}, A\right),\left(F_{2}, A\right)$ as $F_{1}(e)=X$ for $e \in G_{1}, F_{1}(e)=\emptyset$ otherwise and $F_{2}(e)=X$ for $e \in G_{2}, F_{2}(e)=\emptyset$ otherwise. It is clear $e_{F} \tilde{\in}\left(F_{1}, A\right),(G, A) \tilde{\subset}\left(F_{2}, A\right)$ and $\left(F_{1}, A\right) \tilde{\cap}\left(F_{2}, A\right)=\Phi_{A}$. So $(X, \tau, A)$ is soft regular ${ }^{H}$.

Theorem 2.2. Let $(X, \tau, A)$ be a soft topological space. Then it is soft regular $^{S}$ if and only if for any soft closed set $(G, A), \operatorname{Gr}(G)=A \times \operatorname{Ran}(G)$ and a topological space $\left(X, \nu_{\tau}\right)$ is regular, where $\nu_{\tau}=\{\operatorname{Ran}(H):(H, A) \in \tau\}$.

Proof. " $\Rightarrow$ "Suppose $(X, \tau, A)$ is soft $\operatorname{regular}^{S}$ and $(G, A)$ is soft closed. If $x \in G\left(e_{0}\right)$ for some $e_{0} \in A$, then $x \in(G, A)$. Suppose $x \notin(G, A)$. Since $(X, \tau, A)$ is soft regular ${ }^{S}$, there exist soft open sets $\left(F_{1}, A\right),\left(F_{2}, A\right)$ such that $x \in\left(F_{1}, A\right),(G, A) \tilde{\subset}\left(F_{2}, A\right)$ and $\left(F_{1}, A\right) \tilde{\cap}\left(F_{2}, A\right)=\Phi_{A}$, a contradiction with $x \in F_{1}\left(e_{0}\right) \cap F_{2}\left(e_{0}\right)$. That means $G r(G)=A \times \operatorname{Ran}(G)$. Consequently, if $(F, A)$ is soft open, then $\operatorname{Gr}(F)=A \times \operatorname{Ran}(F)$ and $\nu_{\tau}$ is a topology on $A$.

$" \Leftarrow "$ Let $(G, A)$ be a soft closed set and $x \notin(G, A)$. Since $G r(G)=$ $A \times \operatorname{Ran}(G), \operatorname{Ran}(G)$ is closed and $x \notin \operatorname{Ran}(G)$. Then there are disjoint open sets $G_{1}, G_{2} \in \nu_{\tau}$ such that $x \in G_{1}$ and $\operatorname{Ran}(G) \subset G_{2}$. Define $\left(F_{1}, A\right),\left(F_{2}, A\right)$ as $F_{1}(e)=G_{1}$ for any $e \in A$ and $F_{2}(e)=G_{2}$ for any $e \in A$. It is clear $x \in\left(F_{1}, A\right)$, $(G, A) \tilde{\subset}\left(F_{2}, A\right)$ and $\left(F_{1}, A\right) \tilde{\cap}\left(F_{2}, A\right)=\Phi_{A}$. So $(X, \tau, A)$ is soft regular ${ }^{S}$.

The next theorem follows from the previous ones.

Theorem 2.3. Soft topological space $(X, \tau, A)$ is soft regular ${ }^{H}$ (soft regular $\left.^{S}\right)$ if and only if the corresponding topological space $\left(A \times X, \tau_{A \times X}\right)$ is 
homeomorphic to the Cartesian product of a regular (an indiscrete) topological space $A$ and an indiscrete (a regular) topological space $X$.

Definition 2.2. A soft topological space $(X, \tau, A)$ is soft- $T_{1}^{H}[5]$ if for each soft points $e_{F}$ and $e_{G}$ such that $F(e) \cap G(e)=\emptyset$ there exist soft open sets $\left(F_{1}, A\right),\left(F_{2}, A\right)$ such that $e_{F} \tilde{\in}\left(F_{1}, A\right), e_{G} \tilde{\notin}\left(F_{1}, A\right)$ and $e_{G} \tilde{\in}\left(F_{2}, A\right), e_{F} \tilde{\notin}\left(F_{2}, A\right)$.

It is clear that $(X, \tau, A)$ is soft- $T_{1}^{H}$ if and only if $\left(X, t_{e}\right)$ is a $T_{1}$-space for any $e \in A$.

In [5] a soft- $T_{3}^{H}$ separation axiom was introduced. Namely, a soft topological space is soft- $T_{3}^{H}$ if it is soft- $T_{1}^{H}$ and soft regular ${ }^{H}$. By Theorem 2.1, we have the next theorem.

Theorem 2.4. Let $(X, \tau, A)$ be soft topological space. Then it is soft- $T_{3}^{H}$ if and only if $X$ is a singleton.

From the proven results we can see that non-systemic approaches are still emerging in the soft separation axiom setting.

\section{References}

[1] A. Aygünoğlu, H. Aygün, Some notes on soft topological spaces, Neural. Comput. Appl., 21 (2011), 113-119.

[2] N. Çă̆man, S. Karataş, S. Enginoglu, Soft topology, Comput. Math. Appl., 62 (2011), 351-358.

[3] D. N. Georgiou, A. C. Megaritis, Soft set theory and topology, Appl. Gen. Topol., 15, No. 1 (2014), 93-109.

[4] D. N. Georgiou, A. C. Megaritis, V. I. Petropoulos, On Soft Topological Spaces, Appl. Math. Inf. Sci., 7, No. 5 (2013), 1889-1901.

[5] S. Hussain, B. Ahmad, Soft separation axioms in soft topological spaces, Hacettepe Journal of Math. and Statistics, 44, No. 3 (2015), 559-568.

[6] M. Matejdes, Soft topological questions and answers, Int. J. Pure and Appl. Math, 104, No. 2 (2015), 237-247.

[7] M. Matejdes, Soft topological space and topology on the Cartesian product, Hacettepe J. Math. and Statistics, 45, No. 4 (2016), 1091-1100.

[8] W. K. Min, A note on soft topological spaces, Comput. Math. Appl., 62 (2011), 35243528.

[9] M. Shabir, M. Naz, On soft topological spaces, Comput. Math. Appl., 61 (2011), 17861799 . 